\title{
Generalized Horadam Polynomials and Numbers
}

\author{
Snezana S. Djordjevic and Gospava B. Djordjevic
}

\begin{abstract}
We consider the polynomials $h_{n, m}(x)(m \geq 2)$ and the numbers $h_{n, m}$ $(x=1)$, which are the generalized Horadam polynomials and the generalized Horadam numbers, respectively. We also consider the polynomials $h_{n, m}^{(s)}(x)$ - convolutions of the polynomials $h_{n, m}(x)$, and the sequence of numbers $h_{n, m^{-}}^{(s)}$ convolutions of the numbers $h_{n, m}$, where $s \geq 0$.
\end{abstract}

\section{Introduction}

In the paper [8] authors considered Horadam polynomials $h_{n}(x)$, which are given by the following recurrence relation

$$
h_{n}(x)=p x h_{n-1}(x)+q h_{n-2}(x), \quad n>2,
$$

with $h_{1}(x)=a, h_{2}(x)=b x,(a, b, p, q$ are some real constants).

We emphasize some particular cases of the polynomials $h_{n}(x)$ :

$1^{\circ}$ For $a=b=p=q=1$, we get the Fibonacci polynomials $F_{n}(x)$;

$2^{\circ}$ For $a=2, b=p=q=1$, we get the Lucas polynomials $L_{n}(x)$;

$3^{\circ}$ If $a=q=1, b=p=2$, then we get the Pell polynomials $P_{n}(x)$;

$4^{\circ}$ If $a=1, b=p=2, q=-1$, then we get the Chebyshev polynomials of the second kind $U_{n}(x)$.

Key Words: Generalized Horadam polynomials and numbers, Convolution, Recurrence relation; Explicit representation, Generating function.

2010 Mathematics Subject Classification: Primary 11B83; Secondary 11B37, $11 B 39$.

Received: 28.03.2017

Revised: 02.09.2017

Accepted: 05.09.2017 


\section{Generalized polynomials}

In this section we introduce the polynomials $h_{n, m}(x)(m \geq 2)$, the generalized Horadam polynomials, by

$$
h_{n, m}(x)=p x h_{n-1, m}(x)+q h_{n-m, m}(x), \quad n>m,
$$

with $h_{1, m}(x)=a, h_{n, m}(x)=b p^{n-2} x^{n-1}$, for $n=2, \ldots, m$.

For $x=1$ in (2), we obtain the generalized Horadam numbers $h_{n, m}$ :

$$
h_{n, m}=p h_{n-1, m}+q h_{n-m, m}, \quad n>m,
$$

with $h_{1, m}=a, h_{n, m}=b p^{n-2}$, for $n=2, \ldots, m$.

Remark 1. For $m=2$ in (2) and (3), we get Horadam polynomials $h_{n}(x)$ and Horadam numbers $h_{n}$, respectively (see [8]).

Now, using the standard method, starting with the recurrence relation (2), we find that the function

$$
F(x, t)=\frac{a+x t(b-a p)}{1-p x t-q t^{m}}=\sum_{n=1}^{\infty} h_{n, m}(x) t^{n-1}
$$

is the generating function of the polynomials $h_{n, m}(x)$.

Remark 2. For $m=2$, the relation (4) yields the generating function $g(x, t)$ of the Horadam polynomials $h_{n}(x)$ ( see [8], (13)):

$$
g(x, t)=\frac{a+x t(b-a p)}{1-p x t-q t^{2}}=\sum_{n=1}^{\infty} h_{n}(x) t^{n-1} .
$$

Using the development of the function $F(x, t)$, given by (4), into the series on $t$, then comparing the corresponding coefficients to $t^{n}$, we obtain the explicit formula for the polynomials $h_{n, m}(x)$

$$
\begin{aligned}
h_{n, m}(x)= & a \sum_{k=0}^{[(n-1) / m]}\left(\begin{array}{c}
n-1-(m-1) k \\
k
\end{array}\right)(p x)^{n-1-m k} q^{k} \\
& +\left(\frac{b}{p}-a\right) \sum_{k=0}^{[(n-2) / m]}\left(\begin{array}{c}
n-2-(m-1) k \\
k
\end{array}\right)(p x)^{n-1-m k} q^{k} .
\end{aligned}
$$

Remark 3. For $m=2$ the formula (2.4) yields the explicit formula for Horadam polynomials $h_{n}(x)$ ( see [8], (16)). 
Taking $x=1$ in (2.4), we get the explicit formula for the generalized Horadam numbers $h_{n, m}$ :

$$
\begin{aligned}
h_{n, m}= & a p^{n-1} \sum_{k=0}^{[(n-1) / m]}\left(\begin{array}{c}
n-1-(m-1) k \\
k
\end{array}\right)\left(\frac{q}{p^{m}}\right)^{k} \\
& +p^{n-2}(b-a p) \sum_{k=0}^{[(n-2) / m]}\left(\begin{array}{c}
n-2-(m-1) k \\
k
\end{array}\right)\left(\frac{q}{p^{m}}\right)^{k} .
\end{aligned}
$$

Some particular cases of the polynomials $h_{n, m}(x)$ (see $\left.[1,3,4,5,6]\right)$ are:

$$
\begin{gathered}
F_{n+1, m}(x)=\sum_{k=0}^{[n / m]}\left(\begin{array}{c}
n-(m-1) k \\
k
\end{array}\right) x^{n-m k}-\text { Fibonacci polynomials } \\
P_{n+1, m}(x)=\sum_{k=0}^{[n / m]}\left(\begin{array}{c}
n-(m-1) k \\
k
\end{array}\right)(2 x)^{n-m k}-\text { Pell polynomials; } \\
J_{n+1, m}(y)=\sum_{k=0}^{[n / m]}\left(\begin{array}{c}
n-(m-1) k \\
k
\end{array}\right)(2 y)^{k}-\text { Jacobsthal polynomials; } \\
U_{n, m}(x)=\sum_{k=0}^{[n / m]}(-1)^{k}\left(\begin{array}{c}
n-(m-1) k \\
k
\end{array}\right)(2 x)^{n-m k}-\text { Chebyshev polynomials. }
\end{gathered}
$$

\section{Some properties}

Theorem 1. The polynomials $h_{n, m}(x)$ satisfy the following relation

$$
\sum_{k=1}^{n-1} h_{k, m}(x)=\frac{h_{n, m}(x)+q \sum_{i=1}^{m-1} h_{n-i, m}(x)-a-x(b-a p)}{p x+q-1} .
$$


Proof. Starting from the recurrence relation (2) and by the corresponding characteristic equation

$$
\lambda^{m}-p x \lambda^{m-1}-q=0,
$$

where $\lambda_{i}, i=1, \ldots, m$ are the solutions of the equation (3.2), we have:

$$
\begin{aligned}
& \sum_{k=1}^{n-1} h_{k, m}(x)=\sum_{k=1}^{n-1}\left(A_{1} \lambda_{1}^{k-1}+A_{2} \lambda_{2}^{k-1}+\cdots+A_{m} \lambda_{m}^{k-1}\right) \\
& =A_{1}\left(1+\lambda_{1}+\lambda_{1}^{2}+\cdots+\lambda_{1}^{n-2}\right) \\
& \quad \quad+A_{2}\left(1+\lambda_{2}+\lambda_{2}^{2}+\cdots+\lambda_{2}^{n-2}\right)+\cdots+ \\
& \quad \quad+A_{m}\left(1+\lambda_{m}+\lambda_{m}^{2}+\cdots+\lambda_{m}^{n-2}\right) \\
& =A_{1} \frac{1-\lambda_{1}^{n-1}}{1-\lambda_{1}}+A_{2} \frac{1-\lambda_{2}^{n-1}}{1-\lambda_{2}}+\cdots+A_{m} \frac{1-\lambda_{m}^{n-1}}{1-\lambda_{m}} \\
& \left.=\frac{1}{\left(1-\lambda_{1}\right)\left(1-\lambda_{2}\right) \cdots\left(1-\lambda_{m}\right)} \times \prod_{i=1, i \neq 2}^{m}\left(1-\lambda_{i}\right)+\cdots+A_{m}\left(1-\lambda_{m}^{n-1}\right) \prod_{i=1}^{m-1}\left(1-\lambda_{i}\right)\right)
\end{aligned}
$$

Using the relations (by (3.2)) $\lambda_{1}+\lambda_{2}+\cdots+\lambda_{m}=p x, \quad \lambda_{1} \lambda_{2}+\lambda_{1} \lambda_{3}+\cdots+\lambda_{m-1} \lambda_{m}=0, \ldots(-1)^{m} \lambda_{1} \lambda_{2} \cdots \lambda_{m}=-q$, we get

$$
\begin{aligned}
& \left(\sum_{i=1}^{m} A_{i}\left(1-\lambda_{i}^{n-1}\right) \prod_{j=1, j \neq i}^{m}\left(1-\lambda_{j}\right)\right) \cdot\left(\left(1-\lambda_{1}\right)\left(1-\lambda_{2}\right) \cdots\left(1-\lambda_{m}\right)\right)^{-1} \\
& =\frac{1}{1-p x-q} \times \\
& \sum_{i=1}^{m} A_{i}\left(1-\lambda_{i}^{n-1}\right)\left(1-\lambda_{i}\left(p x-\lambda_{i}\right)-\lambda_{i}^{2}\left(p x-\lambda_{i}\right)+\cdots+(-1)^{m-1} \lambda_{i}^{m-1}\left(p x-\lambda_{i}\right)\right) \\
& =\frac{1}{1-p x-q}\left(a-a p x+b x-h_{n, m}(x)-q \sum_{i=1}^{m-1} h_{n-i, m}(x)\right)
\end{aligned}
$$

Remark 4. For $m=2$ the formula (5) yields (see [8], (18))

$$
\sum_{k=1}^{n-1} h_{k}(x)=\frac{h_{n}(x)+q h_{n-1}(x)-a-x(b-a p)}{p x+q-1} .
$$


Some special cases of the formula (5) are (see [5]):

$$
\begin{gathered}
\sum_{k=1}^{n-1} F_{k, m}(x)=\frac{F_{n, m}(x)+\sum_{i=1}^{m-1} F_{n-i, m}(x)-1}{x}, \\
F_{n, m}(x)-\text { the generalized Fibonacci polynomials; } \\
\sum_{k=1}^{n-1} P_{k, m}(x)=\frac{P_{n, m}(x)+\sum_{i=1}^{m-1} P_{n-i, m}(x)-1}{2 x}, \\
P_{n, m}(x)-\text { the generalized Pell polynomials; } \\
\sum_{k=1}^{n-1} U_{k, m}(x)=\frac{U_{n, m}(x)-\sum_{i=1}^{m-1} U_{n-i, m}(x)-1}{2 x-2}
\end{gathered}
$$

$U_{n, m}(x)$ - the generalized Chebyshev polynomials of the second kind.

\section{Convolutions of the generalized Horadam polynomials}

In this section we introduce the polynomials $h_{n, m}^{(s)}(x)$, the convolutions of the polynomials $h_{n, m}(x)$, by

$$
G(x, t)=\left(\frac{a+x t(b-a p)}{1-p x t-q t^{m}}\right)^{s+1}=\sum_{n=1}^{\infty} h_{n, m}^{(s)}(x) t^{n-1},
$$

where $n, m \in \mathbb{N}, s \in \mathbb{N} \cup\{0\}, n \geq m$.

Starting from (7), we get the following representation of the polynomials $h_{n, m}^{(s)}(x)$ :

$$
\begin{aligned}
h_{n, m}^{(s)}(x) & =\sum_{i=0}^{s+1}\left(\begin{array}{c}
s+1 \\
i
\end{array}\right) a^{s+1-i}\left(\frac{b}{p}-a\right)^{i} \\
& \times \sum_{k=0}^{[(n-1) / m]} \frac{(s+1)_{n-1-i-(m-1) k}}{k !(n-1-i-m k) !}(p x)^{n-1-m k} q^{k} .
\end{aligned}
$$

where $\left(\begin{array}{l}n \\ k\end{array}\right)=0$ for $n<k$. 
Some particular cases of the polynomials $h_{n, m}^{(s)}(x)$ are:

$1^{\circ}$ For $a=b=p=q=1$, we have $F_{n, m}^{(s)}(x)$, the convolutions of the generalized Fibonacci polynomials and (4.2) becomes

$$
F_{n, m}^{(s)}(x)=\sum_{k=0}^{[(n-1) / m]} \frac{(s+1)_{n-1-(m-1) k}}{k !(n-1-m k) !} x^{n-1-m k} .
$$

If we use the known relations $([7],[4])$ :

$$
(\alpha)_{n+k}=(\alpha)_{n}(\alpha+n)_{k} ; \frac{(-1)^{k}}{(n-k) !}=\frac{(-n)_{k}}{n !},(\alpha)_{n-k}=\frac{(-1)^{k}(\alpha)_{n}}{(1-\alpha-n)_{k}}
$$

then (4.3) takes the following hypergeometric representation

$$
F_{n+1, m}^{(s)}(x)=\frac{x^{n}(s+1)^{n}}{n !}{ }_{m} F_{m-1}\left[\frac{\frac{-n}{m}, \frac{1-n}{m}, \ldots, \frac{m-1-n}{m} ;-x^{-m}}{\frac{-s-n}{m-1}, \frac{1-s-n}{m-1}, \ldots, \frac{m-2-s-n}{m-1}}\right] .
$$

$2^{\circ}$ For $a=q=1, b=p=2$, we obtain $P_{n, m}^{(s)}(x)$, the convolutions of the generalized Pell polynomials

$$
P_{n+1, m}^{(s)}(x)=\sum_{k=0}^{[n / m]} \frac{(s+1)_{n-(m-1) k}}{k !(n-m k) !}(2 x)^{n-m k},
$$

or

$$
P_{n+1, m}^{(s)}(x)=\frac{(2 x)^{n}(s+1)_{n}}{n !}{ }_{m} F_{m-1}\left[\begin{array}{l}
\frac{-n}{m}, \frac{1-n}{m}, \ldots, \frac{m-1-n}{m} ;(2 x)^{-m} \\
\frac{-s-n}{m-1}, \frac{1-s-n}{m-1}, \ldots, \frac{m-2-s-n}{m-1}
\end{array}\right] .
$$

$3^{\circ}$ For $a=1, b=p=2, q=-1$, in $(4.2)$, we get $U_{n, m}^{(s)}(x)$, the convolutions of the generalized Chebyshev polynomials

$$
U_{n, m}^{(s)}(x)=\frac{(2 x)^{n}(s+1)_{n}}{n !}{ }_{m} F_{m-1}\left[\begin{array}{c}
\frac{-n}{m}, \frac{1-n}{m}, \ldots, \frac{m-1-n}{m} ;(2 x)^{-m} \\
\frac{-s-n}{m-1}, \frac{1-s-n}{m-1}, \ldots, \frac{m-2-s-n}{m-1}
\end{array}\right] .
$$

Next, for $b=a p$ in (4), we get

$$
F(x, t)=a\left(1-p t-q t^{m}\right)^{-1}=\sum_{n=1}^{\infty} h_{n, m}(x) t^{n-1} .
$$

Differentiating both sides of (4.3) to $x$, one by one, $s$-times, we find that

$$
h_{n-s, m}^{(s)}(x)=\frac{1}{a p^{s} s !} D^{s} h_{n, m}(x), \text { where } h_{n, m}^{s}(x) \equiv \frac{\partial^{s} h_{n, m}(x)}{\partial x^{s}} .
$$

Namely, using the formula (4.4), we easily calculate the convolutions of the polynomials $h_{n, m}^{(s)}(x)$. Next, we give the examples for $m=3$ and $s=0,1,2,3$, and for $m=4$ and $s=0,1,2,3$. 
Table 1: $h_{n, 3}^{(s)}(x)$

\begin{tabular}{||l|l||l||}
\hline \hline$n$ & $s=0$ & $s=1$ \\
\hline 1 & $a$ & 1 \\
\hline 2 & $a p x$ & $2 p x$ \\
\hline 3 & $a(p x)^{2}$ & $3(p x)^{2}$ \\
\hline 4 & $a(p x)^{3}+a q$ & $4(p x)^{3}+2 q$ \\
\hline 5 & $a(p x)^{4}+2 a p q x$ & $5(p x)^{4}+6 p q x$ \\
\hline 6 & $a(p x)^{5}+3 a q(p x)^{2}$ & $6(p x)^{5}+12 q(p x)^{2}$ \\
\hline 7 & $a(p x)^{6}+4 a q(p x)^{3}+a q^{2}$ & $7(p x)^{6}+20 q(p x)^{3}+3 q^{2}$ \\
\hline 8 & $a(p x)^{7}+5 a q(p x)^{4}+3 a q^{2} p x$ & $8(p x)^{7}+30 q(p x)^{4}+12 q^{2} p x$ \\
\hline 9 & $a(p x)^{8}+6 a q(p x)^{5}+6 a q^{2}(p x)^{2}$ & $9(p x)^{8}+42 q(p x)^{5}+30 q^{2}(p x)^{2}$ \\
\hline \hline
\end{tabular}

Table 2: $h_{n, 3}^{(s)}(x)$

\begin{tabular}{||l|l||l||}
\hline \hline$n$ & $s=2$ & $s=3$ \\
\hline 1 & 1 & 1 \\
\hline 2 & $3 p x$ & $4 p x$ \\
\hline 3 & $6(p x)^{2}$ & $10(p x)^{2}$ \\
\hline 4 & $10(p x)^{3}+3 q$ & $20(p x)^{3}+4 q$ \\
\hline 5 & $15(p x)^{4}+12 p q x$ & $35(p x)^{4}+20 p q x$ \\
\hline 6 & $21(p x)^{5}+30 q(p x)^{2}$ & $56(p x)^{5}+60 q(p x)^{2}$ \\
\hline 7 & $28(p x)^{6}+60 q(p x)^{3}+6 q^{2}$ & $84(p x)^{6}+140 q(p x)^{3}+10 q^{2}$ \\
\hline 8 & $36(p x)^{7}+105 q(p x)^{4}+30 q^{2} p x$ & $120(p x)^{7}+280 q(p x)^{4}+60 q^{2} p x$ \\
\hline 9 & $45(p x)^{8}+168 q(p x)^{5}+90 q^{2}(p x)^{2}$ & $165(p x)^{8}+504 q(p x)^{5}+210 q^{2}(p x)^{2}$ \\
\hline \hline
\end{tabular}


Table 3: $h_{n, 4}^{(s)}(x)$

\begin{tabular}{||l|l||l||}
\hline \hline$n$ & $s=0$ & $s=1$ \\
\hline 1 & $a$ & 1 \\
\hline 2 & $a p x$ & $2 p x$ \\
\hline 3 & $a(p x)^{2}$ & $3(p x)^{2}$ \\
\hline 4 & $a(p x)^{3}$ & $4(p x)^{3}$ \\
\hline 5 & $a(p x)^{4}+a q$ & $5(p x)^{4}+2 q$ \\
\hline 6 & $a(p x)^{5}+2 a q p x$ & $6(p x)^{5}+6 q p x$ \\
\hline 7 & $a(p x)^{6}+3 a q(p x)^{2}$ & $7(p x)^{6}+12 q(p x)^{2}$ \\
\hline 8 & $a(p x)^{7}+4 a q(p x)^{3}$ & $8(p x)^{7}+20 q(p x)^{3}$ \\
\hline 9 & $a(p x)^{8}+5 a q(p x)^{4}+a q^{2}$ & $9(p x)^{8}+30 q(p x)^{4}+3 q^{2}$ \\
\hline \hline
\end{tabular}

Table 4: $h_{n, 4}^{(s)}(x)$

\begin{tabular}{||c|l||l||}
\hline \hline$n$ & $s=2$ & $s=3$ \\
\hline 1 & 1 & 1 \\
\hline 2 & $3 p x$ & $4 p x$ \\
\hline 3 & $6(p x)^{2}$ & $10(p x)^{2}$ \\
\hline 4 & $10(p x)^{3}$ & $20(p x)^{3}$ \\
\hline 5 & $15(p x)^{4}+3 q$ & $35(p x)^{4}+4 q$ \\
\hline 6 & $21(p x)^{5}+12 q p x$ & $56(p x)^{5}+20 q p x$ \\
\hline 7 & $28(p x)^{6}+30 q(p x)^{2}$ & $84(p x)^{6}+60 q(p x)^{2}$ \\
\hline 8 & $36(p x)^{7}+60 q(p x)^{3}$ & $120(p x)^{7}+140 q(p x)^{3}$ \\
\hline 9 & $45(p x)^{8}+105 q(p x)^{4}+6 q^{2}$ & $165(p x)^{8}+56 q(p x)^{4}+10 q^{2}$ \\
\hline \hline
\end{tabular}


Table 5: $P_{n, 4}^{(s)}(x)$-Pell polynomials

\begin{tabular}{||l|l||l||}
\hline \hline$n$ & $s=0$ & $s=1$ \\
\hline 1 & 1 & 1 \\
\hline 2 & $2 x$ & $2(2 x)$ \\
\hline 3 & $(2 x)^{2}$ & $3(2 x)^{2}$ \\
\hline 4 & $(2 x)^{3}$ & $4(2 x)^{3}$ \\
\hline 5 & $(2 x)^{4}+1$ & $5(2 x)^{4}+2$ \\
\hline 6 & $(2 x)^{5}+2(2 x)$ & $6(2 x)^{5}+6(2 x)$ \\
\hline 7 & $(2 x)^{6}+3(2 x)^{2}$ & $7(2 x)^{6}+12(2 x)^{2}$ \\
\hline 8 & $(2 x)^{7}+4(2 x)^{3}$ & $8(2 x)^{7}+20(2 x)^{3}$ \\
\hline 9 & $(2 x)^{8}+5(2 x)^{4}+1$ & $9(2 x)^{8}+30(2 x)^{4}+3$ \\
\hline \hline
\end{tabular}

Table 6: $P_{n, 4}^{(s)}(x)$

\begin{tabular}{||c|l||l||}
\hline \hline$n$ & $s=2$ & $s=3$ \\
\hline 1 & 1 & 1 \\
\hline 2 & $3(2 x)$ & $4(2 x)$ \\
\hline 3 & $6(2 x)^{2}$ & $10(2 x)^{2}$ \\
\hline 4 & $10(2 x)^{3}$ & $20(2 x)^{3}$ \\
\hline 5 & $15(2 x)^{4}+3$ & $35(2 x)^{4}+4$ \\
\hline 6 & $21(2 x)^{5}+12(2 x)$ & $56(2 x)^{5}+20(2 x)$ \\
\hline 7 & $28(2 x)^{6}+30(2 x)^{2}$ & $84(2 x)^{6}+60(2 x)^{2}$ \\
\hline 8 & $36(2 x)^{7}+60(2 x)^{3}$ & $120(2 x)^{7}+140(2 x)^{3}$ \\
\hline 9 & $45(2 x)^{8}+105(2 x)^{4}+6$ & $165(2 x)^{8}+56(2 x)^{4}+10$ \\
\hline \hline
\end{tabular}


Table 7: $P_{n, 4}^{(s)}$-Pell numbers

\begin{tabular}{||l||l||l||l||l||}
\hline \hline$n$ & $s=0$ & $s=1$ & $s=2$ & $s=3$ \\
\hline 1 & 1 & 1 & 1 & 1 \\
\hline 2 & 2 & 4 & 6 & 8 \\
\hline 3 & 4 & 12 & 24 & 40 \\
\hline 4 & 8 & 32 & 80 & 160 \\
\hline 5 & 17 & 82 & 243 & 564 \\
\hline 6 & 36 & 204 & 696 & 1832 \\
\hline 7 & 76 & 7496 & 1912 & 5616 \\
\hline 8 & 160 & 1184 & 4608 & 16480 \\
\hline 9 & 337 & 2787 & 13206 & 43146 \\
\hline \hline
\end{tabular}

\section{References}

[1] G. B. Djordjevic, Some generalization of the Jacobsthal numbers, Filomat, 24(2)(2010), 143-151.

[2] G. B. Djordjevic, Generalization of the Fibonacci and Lucas polynomials, Filomat, 23(3)(2009), 291-301.

[3] G. B. Djordjevic, H. M. Srivastava, Some generalization of the incomplete Fibonacci and incomplete Lucas polynomials, Adv. Stud. Contemp. Math. 11(2005), 11-32.

[4] G. B. Djordjevic, Convolutions of the generalized Pell and Pell-Lucas numbers, Filomat, 30(1)(2016), 105-112.

[5] G. B. Djordjevic, Polynomials related to generalizede Chebyshev polynomils, Filomat, 23(3)(2009), 279-290.

[6] A. F. Horadam, Convolution summations for Pell and Pell-Lucas numbers, The Fibonacci Quart. 38(5)(2000), 451-457.

[7] E. D. Rainville, Special functions, The Macmillan Company, New York, 1960.

[8] Tugba Horcum, E. Gokcen Kocer, On some properties of Horadam polynomials, Int. Math. Forum, 4(25)(2009), 1243-1252. 
Snezana S. Djordjevic,

Faculty of technology, Department of Mathematics,

University of Nis,

Bulevar Oslobodjenja 124, 16000 Leskovac, Serbia.

Email: snezanadjordjevic1971@gmail.com

Gospava B. Djordjevic,

Faculty of technology, Department of Mathematics,

University of Nis,

Bulevar Oslobodjenja 124, 16000 Leskovac, Serbia.

Email: gospava48@ptt.rs 